\title{
LA TEORIA DELLA GIUSTIZIA DI NANCY FRASER COME CONTRIBUTO ALL'ANALISI DELLE POLITICHE DI AZIONE AFFERMATIVA NELL'ISTRUZIONE SUPERIORE BRASILIANA
}

\section{ARTICOLO ORIGINALE}

GUIMARÃES, Matheus De Oliveira ${ }^{1}$

GUIMARÃES, Matheus De Oliveira. La teoria della giustizia di Nancy Fraser come contributo all'analisi delle politiche di azione affermativa nell'istruzione superiore brasiliana. Revista Científica Multidisciplinar Núcleo do Conhecimento. anno 04, Ed. 11, Vol. 01, pp. 22-38. novembre 2019. ISSN: 2448-0959, Collegamento di accesso: https://www.nucleodoconhecimento.com.br/formazione-it/teoria-dellagiustizia

\section{RIEPILOGO}

Questo articolo cerca la presentazione della formulazione della giustizia sociale nominata dalla filosofa politica americana Nancy Fraser attraverso la quale interpreta lo scenario sociale oggi dal riconoscimento binomiale-ridistribuzione. Presenteremo argomenti teorici che possono essere di valore interessante per l'analisi, l'interpretazione e la valutazione delle politiche educative brasiliane direttamente legate al tema delle politiche di azione affermativa. Dalla presentazione della teoria frasene sulla giustizia sociale e il riconoscimento, cerchiamo di mettere in relazione questo pensiero, in modo panoramico, con le azioni affermative volte all'istruzione superiore attuata in Brasile - in particolare dal decennio del 1990. A tal fine, vengono

\footnotetext{
${ }^{1}$ Dottorato di ricerca in Diritto all'istruzione e politiche educative del Graduate Program in Education della Pontificia Università Cattolica di Minas Gerais (PucMinas). Laurea magistrale in Educazione, legata alla linea di ricerca Diversità, Inclusione e Pratiche Educative, dell'Università Federale di Ouro Preto (UFOP). Ha conseguito una laurea in Giurisprudenza (UFOP), Filosofia (ICSH) e Pedagogia (FGD), specializzandosi in Pratiche Pedagogiche (UFOP) e Public Policy Management (UFOP).
} 
dimostrate le principali argomentazioni di Nancy fraser, per le quali oggi la giustizia richiede sia la ridistribuzione dei beni sociali e della ricchezza e il riconoscimento culturale-valorativo delle differenze. Infine, viene stabilito il rapporto teorico tra azioni affermative e teoria frasene.

Parole chiave: azioni affermative, istruzione superiore, giustizia sociale, Nancy Fraser, razza, riconoscimento della ridistribuzione.

\section{INTRODUZIONE}

La Costituzione brasiliana del 1988, nel suo articolo 206, l'articolo I, e la legge brasiliana sulle linee guida e le basi dell'istruzione del 1996, nell'articolo 3, punto I, stabiliscono che l'insegnamento deve essere praticato nel rispetto del principio di parità di condizioni. In linea con questo precetto costituzionale, le politiche educative cominciarono ad essere formulate e attuate con lo scopo di ridurre le disuguaglianze sociali che hanno prevalso nella storia del Brasile - discriminando i vari segmenti della società.

Negli ultimi due decenni, per quanto riguarda il trattamento del tema razziale in Brasile, si sono verificati cambiamenti significativi nel paese che hanno prodotto intense trasformazioni nella società. In questo contesto, sono incluse azioni affermative, intese come iniziative per promuovere l'inclusione sociale e, allo stesso tempo, vengono valutati aspetti singolari dei gruppi e degli individui.

Il collegamento tra le discussioni di Educazione, Diritto e Scienze Politiche con temi legati a questioni di ridistribuzione e riconoscimento - e in particolare per quanto riguarda la razza - si basa in modo significativo sulla sua indiscutibile enfasi sulla comprensione e far fronte alle disuguaglianze nel contesto sociale, sia nelle relazioni di potere che nella conoscenza, ineguagliabilmente interconnesse (APPLE; PALLA; GANDIN, 2013; GILLBORN; LADSON-BILLINGS, 2013; APPLE, 2017). Nonostante la comprensione da parte di molti di un presunto carattere epifenomenale di ciò che alla fine riguarderebbe le discussioni sulla classe e sul capitalismo, è essenziale comprendere la forza relativamente autonoma delle questioni razziali e l'importanza di 
concentrarsi sulle particolarità della vita quotidiana di coloro che vivono tali situazioni (APPLE, 2017).

In questo senso, con la necessità di fornire l'accesso (e la permanenza) all'istruzione superiore a coloro che hanno trascurato le loro possibilità di istruzione, le politiche pubbliche brasiliane hanno cercato una tardiva universalizzazione dell'istruzione superiore $e$, forse nonostante l'intenzione, finiscono in linea con la perdita della qualità dell'insegnamento senza democratizzare efficacemente l'accesso. Sebbene negli ultimi due decenni gli indicatori educativi siano migliorati per tutte le gamme di insegnamento e in tutti i gruppi sociali, si osservano ancora gravi disuguaglianze razziali, soprattutto nelle prime fasi (HEILBORN; L'AA-JO; BARRETO, 2010).

Nonostante il dibattito sulle politiche di azione affermativa si sia concentrato, soprattutto dall'ultimo decennio, fortemente sul sistema delle quote per le università pubbliche, le azioni dello Stato comportano azioni che vanno oltre le quote - con caratteristiche e un ambito differenziato.

Anche se è possibile affermare che nel governo Fernando Henrique Cardoso c'erano già iniziative federali rivolte alla popolazione nera, l'analisi dei documenti del periodo rivela che la strategia discorsiva e la politica di tale governo era quella di promuovere il riconoscimento senza investimenti nell'aspetto redistributivo, anche se la disuguaglianza era la principale giustificazione delle politiche di valorizzamento della popolazione nera (LIMA, 2010).

L'inizio dell'amministrazione di Lula nel 2003 segna un fitto cambiamento delle politiche con una prospettiva razziale. Come afferma Lima (2010), fino ad allora il rapporto dello Stato con il Movimento Nero era di esteriorità, fondamentalmente che si occupava di attori sociali come querelanti e con poco inserimento nell'apparato governativo. Nel governo di lula, questo rapporto deriva dall'incorporazione, nei suoi quadri, di rappresentanti neri (dando maggiore visibilità alle esigenze del movimento).

Il fatto è che il tema dell'istruzione superiore è certamente quello che mobilita il dibattito più pubblico sulle azioni affermative, in particolare per quanto riguarda l'attuazione 
delle politiche di contingenziale negli istituti pubblici di istruzione superiore e le analisi sugli studenti detentori di quote. Tuttavia, come afferma Lima (2010), la marcata reazione sociale alla politica delle quote non ha avuto la stessa ripercussione quando il governo ha attuato un programma di azioni affermative nel sistema privato responsabile di oltre l' $80 \%$ dell'iscrizione all'istruzione superiore brasiliana .

Le principali politiche pubbliche della portata federale con il taglio razziale nell'istruzione sono oggi organizzate sia nell'ambito del riconoscimento culturale che in quello della ridistribuzione. In Brasile, oltre alla famigerata politica delle quote (spesso erroneamente intesa come sintesi di azioni affermative), PROUNI (University For All Program), attraverso la quale il governo federale offre borse di studio (integrali e per gli studenti a basso reddito in modo che possano studiare in college privati; (Fondo di finanziamento degli studenti), un programma del Ministero dell'Istruzione volto a finanziare l'istruzione universitaria degli studenti iscritti a istituti non liberi; e il PBP (Permanence Scholarship Program), un aiuto finanziario (offerto dal governo federale agli studenti a basso reddito nelle università pubbliche federali e agli studenti con borse di studio complete di PROUNI) che mira a ridurre al minimo le disuguaglianze sociali e contribuire alla permanenza e alla laurea degli studenti universitari in una situazione di vulnerabilità socioeconomica.

Di queste politiche, Prouni è, naturalmente, l'azione affermativa più redistributiva di maggiore impatto nella storia dell'istruzione superiore nel paese. Creato nel 2004, sotto Lula, da Provisional Measure 213/2004, Prouni è stato istituzionalizzato nel 2005 dalla legge 11.096/2005. Questo programma mira a concedere borse di studio complete e parziali agli studenti a basso reddito in corsi di laurea e sequenziali di formazione specifica in istituti privati di istruzione superiore. Le esenzioni fiscali sono offerte agli istituti anuouli al Programma.

Corroborando l'idea di una democratizzazione troncata dell'accesso all'istruzione superiore da azioni affermative, Pereira prevede, riferendosi indirettamente ai risultati di Prouni: 
[...] il settore privato in Brasi[responde]le per l'88\% dell'offerta di posti vacanti e per il $71 \%$ di iscrizione, rendendolo uno dei più grandi al mond[...]o. Con l'aiuto della rete privata, l'offerta di posti vacanti per l'istruzione superiore oggi copre l' $86 \%$ dei diplomati delle scuole superiori. [Contudo][...]Solo il 10\% dei giovani di diciotto-ventiquattro anni vi partecipa (2009, p. 45).

Pereira (2009) aggiunge che i posti vacanti inattivi nell'iPA privato erano, prima dell'attuazione di Prouni, al 49,5\% - mentre negli anni 'IT federale questo tasso era dello $0,9 \%$ e, nello stato, del $4,7 \%$.

Sulle politiche di espansione dell'istruzione superiore, Pereira informa:

Lo scenario economico che stiamo vivendo sta dimostrando che gli effetti della democratizzazione educativa non portano alla socialdemocratizzazione, ma che possono produrre nuove e più dolorose disuguaglianze. (2009, p. 46).

Ed è proprio come una proposta per lo studio di questo complesso contesto che indica I'analisi delle teorizations presentate da Nancy Fraser $(2008,2003,2002,2000,1997)$. Le azioni affermative volte a democratizzare l'accesso e la permanenza delle minoranze nell'istruzione superiore sarebbero efficaci per la promozione della trasformazione sociale? Da un punto di vista economico, sarebbero gli stessi efficaci? E quali sono i vostri impatti sul campo del riconoscimento culturale-valorizzante del vostro pubblico di destinazione?

Non è inteso, in questo testo, risposte conclusive a queste domande. Tuttavia, si ritiene che la comprensione degli argomenti fraseani in questo contesto sia di valore interessante per questa analisi. 


\section{ECONOMIC-DISTRIBUTIVE POLICIES X CULTURAL POLICIES:}

\section{NANCY FRASER'S THEORIATIONS ON THE DILEMMA REDISTRIBUTION-RECOGNITION AS A SUB ${ }^{[2] S I D Y ~ P E R ~}$ L'INTERPRETAZIONE DELLE POLITICHE DI AZIONE AFFIRMATIVE IN BRASILIAN OCANI-ONER}

Nancy Fraser $(2008,2003,2002,2000,1997)$ indica la lotta per il riconoscimento come qualcosa che diventa, a grandi passi, la forma paradigmatica del conflitto politico dalla fine del secolo scorso. Secondo Fraser $(2008,2003,2002,2000,1997)$, le richieste di riconoscimento della differenza alimentano le lotte di gruppi mobilitati sotto bandiere diverse : nazionalità, etnia, razza, genere e sessualità. Di conseguenza, l'identità di gruppo va oltre, in questi conflitti (chiamati post-socialisti), l'interesse di classe stesso come principale mezzo di mobilitazione poli[a]tica: " dominio culturale supplerilo come ingiustizia fondamentale. E il riconoscimento culturale prende il posto della ridistribuzione socioeconomica come rimedio all'ingiustizia e all'obiettivo della lotta politica" (FRASER, 2008, p.11, traduzione della mia).

Tuttavia, non è tutto - dal momento che le lotte per il riconoscimento si sviluppano in un mondo di esacerbate disuguaglianze materiali - soprattutto nei paesi meridionali.

In questo contesto, nelle sue teorizzazioni, Fraser $(2008,2003,2002,2000,1997)$ solleva alcune questioni che illustreranno il suo dibattito sui dilemmi della giustizia:

Come dovremmo allora vedere l'eclissi di un immaginario socialista centrato su termini come l'interesse, l'esplorazione e la ridistribuzione? E cosa dovremmo fare con l'emergere di un nuovo immaginario politico incentrato sulle nozioni di identità, differenza, dominio culturale $e$ riconoscimento? Questo imorso rappresenta un lasso di falsa coscienza? $\mathrm{O}$, invece, sarebbe un modo per compensare la cecità culturale di un paradigma marxista messo a discreditare dal crollo del comunismo sovietico? (FRASER, 2008, p.11, traduzione della mia, autore griffins).

Categoricamente, Fraser $(2008,2003,2002)$ afferma che nessuna di queste posizioni sarebbe appropriata, caratterizzandole come "troppo complete e senza sfumature". Piuttosto che semplicemente abolire o respingere quella che sarebbe una semplice 
tonnellata nella politica dell'identità, va considerato che ci sia una sfida intellettuale e pratica: quella di sviluppare una teoria critica del riconoscimento che identifichi e assuma la difende solo quelle versioni della politica culturale di differenza che possono essere combinate in modo coerente con la politica di uguaglianza sociale (FRASER, 2008, p. 11-12).

Nella formulazione di questo progetto, Fraser (2008) afferma che la giustizia oggi richiede sia una ridistribuzione che un riconoscimento, proponendo di esaminare il rapporto tra i due. Questo, secondo l'autore, significherebbe, in parte, pensare a come concettualizzare il riconoscimento culturale e l'uguaglianza sociale in modo che entrambi si sostengano - piuttosto che annientarsi (poiché ci sono diverse concezioni concorrenti tra di loro). Inoltre, significherebbe anche teorizzare i mezzi attraverso i quali la privazione economica e la mancanza di rispetto culturale si intrecciano e si sostengono reciprocamente in modo concomitante. In questo senso, inoltre, per l'autore, la giustizia esige anche che i dilemmi politici che si presentano siano chiariti quando cerchiamo di combattere entrambe le ingiustizie allo stesso tempo (FRASER, 2008, p. 12).

Fraser sostiene, come uno dei suoi scopi:

(...) due problemi politici che ora sono dissociati l'uno dall'altro. Solo con l'integrazione tra riconoscimento e ridistribuzione raggiungeremo un quadro concettuale adeguato alle esigenze della nostra epoca (FRASER, 2008, p.12, traduzione della mia).

Due modalità generiche di comprensione dell'ingiustizia sono presentate da Fraser (2008, p. 13-18).

Il primo sarebbe quello che si riferisce all'ingiustizia economica - che, secondo essa, è radicata nella struttura economico-politica della società (sfruttamento, emarginazione economica e privazione) e la cui concettualizzazione della sua natura è già stata intrapresa, attraverso grandi sforzi, da parte di teoristi egualitari (FRASER, 2008, p. 13-14). 
II secondo modo di comprendere l'ingiustizia, secondo Fraser (2008), è culturale o simbolico, approfondire l'ingiustizia nei modelli sociali di rappresentazione, interpretazione e comunicazione - $i$ cui esempi, secondo l'autore, includono la dominazione culturale (per quanto riguarda la sottomissione a modelli di interpretazione e comunicazione associati ad un'altra cultura, non correlata e/o ostile alla propria); occultamento (relativo all'invisibilizazione per effetto delle pratiche comunicative, interpretive e rappresentative accontate dalla cultura stessa); e mancanza di rispetto (caratterizzata da diffamazione e squalifica di routine nelle rappresentazioni culturali pubbliche stereotipate e/o nelle interazioni della vita quotidiana) (FRASER, 2008, p. 14).

Tuttavia, nonostante le distinzioni tra queste due forme di ingiustizia (socioeconomica e culturale), Fraser $(2008,2003,2002,2000)$ afferma che entrambe sono notevolmente presenti nelle società contemporanee. Entrambi sono radicati in processi e pratiche che promuovono svantaggi ad alcuni gruppi rispetto ad altri. In questo senso, sia l'una che l'altra forma di ingiustizia deve essere sastata- anche se in modi diversi.

Per Fraser (2008, 2003, 2002, 2000), il rimedio all'ingiustizia economica sarebbe una sorta di ristrutturazione politico-economica che potrebbe comportare la ridistribuzione del reddito, la riorganizzazione della divisione del lavoro, i controlli democratici degli investimenti o trasformazione di altre strutture economiche di base. II filosofo politico attribuisce a questo insieme di rimedi per l'ingiustizia economica il termine generico ridistribuzione.

Al contrario, secondo l'autore, il rimedio all'ingiustizia culturale sarebbe una sorta di cambiamento culturale o simbolico. Ciò potrebbe comportare la valorizzazione delle identità sconsiderate (così come i prodotti culturali dei gruppi difamati), l'apprezzamento positivo della diversità culturale e, più radicalmente, potrebbe comprendere una trasformazione globale degli standard sociali rappresentazione, interpretazione e comunicazione, al fine di trasformare il significato del sé e di tutte le persone. Fraser $(2008,2003,2002,2000)$ dà a questa serie di rimedi per l'ingiustizia culturale il termine riconoscimento generico. 
Per l'autore (FRASER, 2008), quando si tratta di collettività che si avvicinano al tipo ideale della classe sfruttata, si trovano ad affrontare ingiustizie distributive che mancano di rimedi redistributivi (la cui logica sarebbe quella di promuovere l'omogeneizzazione e caratterizzazione non corretto dei gruppi). Quando, a sua volta, ci occupiamo di collettività che si avvicinano al tipo ideale di sessualità disprezzata, ad esempio, si affrontano ingiustizie di discriminazione negativa che devono essere rimedi di riconoscimento (la cui logica, a sua volta, sarebbe la valorizzazione del riconoscimento delle loro specificità). Tuttavia, mentre si allontana dalle estremità, le cose diventano sfocate e la differenziazione diventa molto più complessa e delicata. Secondo Fraser (2008), quando si considerano le collettività situate nella regione intermedia dello spettro concettuale, esistono tipi ibridi che mescolano le caratteristiche della classe sfruttata con caratteristiche di sessualità disprezzata. Queste collettività sono chiamate, come prevede l'autore, bivalenti. Essi differiscono in quanto collettività sia a causa della struttura economica politica che della struttura culturale-valorative della società:

Queste collettività bivalenti (oppresse o subordinate) subiscono ingiustizie che contemporaneamente risalgono all'economia politica e alla cultura. In breve, come informa Fraser, queste collettività possono soffrire di scarsa distribuzione socioeconomica e di disprezzo culturale, in modo che nessuna di queste ingiustizie sia di per sé un effetto indiretto dell'altro, ma sia primario che co-originale. In questo caso, non sarebbero sufficienti né i farmaci di ridistribuzione né quelli di riconoscimento da soli. Fraser afferma categoricamente: "collettività bivalente mancano di entrambi"[redistribuição e reconhecimento] (FRASER, 2008, p. 23, la mia traduzione).

A seconda di Fraser, il genere e la razza sono paradigmi di collettività bivalente. Sebbene non abbiano condiviso particolarità tra di loro, sia le dimensioni economiche che quelle culturali-vaorative: "il genere e la razza, quindi, implicano sia la ridistribuzione che il riconoscimento" (FRASER, 2008, p. 23, traduzione della mia).

Così, secondo Fraser (2008, p. 25, la mia traduzione), "la razza [...]è una modalità bivalente di collettività". 
Da un lato, la lotta contro il razzismo assomiglia alla classe, essendo un principio strutturale dell'economia politica. A questo proposito, la razza struttura la divisione capitalista del lavoro. Le occupazioni a basso livello di retribuzione e a basso livello, quelle noiose, sporche e domestiche sono occupate in modo sproporzion ${ }^{[3]}$ ato dai neri, mentre quelle occupazioni meglio retribuite, con uno status più elevato, colletti bianchi, professionisti, tecniche e sono per lo più occupati da bianchi.

Inoltre, secondo l'autore $(2008,2003,2002,2000,1997)$, la razza struttura anche l'accesso al mercato del lavoro formale, costituendo vasti segmenti della popolazione nera come una sottoclasse (ignorata anche per l'azienda stessa) ed esclusa dal sistema Produttivo. Di conseguenza, esiste una struttura politico-economica che genera modalità di sfruttamento, emarginazione e privazione segnate specificamente dalla razza: "questa struttura costituisce la razza come una differenziazione economico-politica conclusa con certi caratteristiche" (2008, p. 26, la mia traduzione).

A questo proposito, secondo l'autore (2008), l'ingiustizia razziale appare come una sorta di ingiustizia distributiva che richiede un risarcimento redistributivo. Così, in modo molto simile alla classe, la giustizia razziale richiede la trasformazione dell'economia politica per eliminare questa razzializzazione. E la logica del rimedio è simile alla logica relativa alla classe: "si tratta di fare gara fuori business" (FRASER, 2008, p. 26, la mia traduzione). Se la razza non è altro che una differenziazione economico-politica, la giustizia richiede, come dice Fraser $(2008,2003,2002,2000)$, che venga abolita come criterio di differenziazione - in questo senso.

Tuttavia, come ha detto l'autore quando si tratta di genere, la razza, così come questo, non è solo economico-politica. La razza ha anche dimensioni culturali-valorative che lo inseriscono nell'universo del riconoscimento, comprendendo anche elementi più simili alla sessualità che con la classe (FRASER, 2008, p. 26).

Come sottolinea Fraser (2008), un aspetto basale del razzismo è l'eurocentrismo caratterizzato dalla costruzione autorizzata di norme che favoriscono i tratti associati alla crusca. A questo si aggiunge il razzismo culturale, caratterizzato, a sua volta, dalla diffusa squalifica delle cose codificate come nero, marrone o giallo (p. 26). 
Come nel caso del genere, questi danni sono ingiustizie di riconoscimento e, pertanto, la logica del rimedio è anche quello di concedere un riconoscimento positivo a un gruppo specificamente svalutato.

Fraser $(2008,2003,2002,2000,1997)$ indica così la corsa come modalità bivalente di collettività, con un volto economico-politico-culturale e altro-vaorative - entrambi i fronti si intrecciano per rafforzarsi a vicenda, dialetticamente - ancor più perché Le norme culturali razziste ed eurocentriche sono istituzionalizzate nello Stato e nell'economia, mentre lo svantaggio economico subito dai neri limita la loro voce.

Per compensare l'ingiustizia razziale, l'economia politica e la cultura si commuovono. Ma, come dimostra l'autore quando si tratta di genere, il carattere bivalente della razza è anche la fonte di un dilemma.

Fraser $(2008,2003,2002,2000)$ afferma che il genere e la razza sono quindi modi dilemmatici di collettività. A differenza della classe, che occupa un'estremità dello spettro concettuale presentato dall'autore, e la sessualità, che occupa l'altra punta, il genere e la razza sono bivalenti, essendo implicati contemporaneamente nella politica di ridistribuzione e nella politica del Riconoscimento.

Ma come si può sciogliere la differenziazione allo stesso tempo e dissolversi la specificità di una collettività disprezzata?

Dopo aver presentato il dilemma della ridistribuzione-riconoscimento in modo apparentemente intrattabile e di assumere che i rimedi redistributivi per l'ingiustizia economico-politica differenziano sempre i gruppi sociali, mentre i rimedi di riconoscimento per l'ingiustizia culturale che mette in evidenza sempre la differenziazione del gruppo sociale, Fraser (2008, p. 28) propone di esaminare concezioni alternative di ridistribuzione - da un lato - e di riconoscimento - dall'altro.

Con rimedi affermativi per l'ingiustizia, Fraser comprende quelli volti a correggere gli effetti disuguali degli accordi sociali senza la struttura sottostante che li genera (2008, p. 28). 
Per rimedi trasformativi, invece, l'autore comprende quelli incentrati sulla correzione degli effetti disuguali rimodellando la struttura gerativa sottostante (2008, p. 28).

In termini di autore, il punto fondamentale di contrasto tra rimedi positivi e rimedi trasformativi per l'ingiustizia è "effetti terminali contro processi che li producono. Non si tratta di un cambiamento graduale rispetto al cambiamento apocalittico" (FRASER, 2008, p. 28, la mia traduzione).

Fraser (2008) spiega questa distinzione, in primo luogo, nel caso di rimedi all'ingiustizia culturale. Secondo l'autore, i rimedi affermativi per tali ingiustizie sono associati a quello che lei chiama "multiculturalismo mainstream" - una sorta di multiculturalismo che propone di compensare la mancanza di rispetto con la rivalutazione delle identità di gruppo ingiustamente svalutato (lasciando intatto il contenuto di queste identità e le differenziazioni di gruppo sottostanti). Al contrario, i rimedi trasformativi sono associati alla decostruzione, compensando la mancanza di rispetto attraverso la trasformazione della struttura culturale-valorosa sottostante. Come afferma Fraser, destabilizzando le identità di gruppo e le differenziazioni esistenti, questi rimedi non solo aumenterebbero l'autostima dei membri di gruppi ancora non rispettati, ma trasformerebbero anche il senso di appartenenza e affiliazione di tutti (p. 28-29).

Per l'autore, il problema non riguarda la dissoluzione di ogni differenza in un'identità umana unica e universale, ma piuttosto il mantenimento di un campo di differenze multiple, non binario, fluido, sempre in movimento:

Mentre i rimedi di riconoscimento affermativo tendono a promuovere le differenziazioni di gruppo esistenti, i rimedi di riconoscimento trasformativo tendono a lungo termine a destabilizzarli al fine di fare spazio a futuri raggruppamenti (FRASER, 2008, p. 30, la mia traduzione).

Distinzioni analoghe si applicano ai rimedi per l'ingiustizia economica. Come dice Fraser $(2008,2003,2002)$, rimedi affermativi per questo tipo di ingiustizia sono storicamente associati allo stato sociale liberale, cercando, attraverso di essi, un risarcimento per la scarsa distribuzione dei terminali (mentre la maggior parte della struttura economico-politica sottostante). I rimedi trasformativi, contro, storicamente si 
associano al socialismo, cercando di compensare la distribuzione sleale attraverso la trasformazione della struttura economico-politica esistente.

Fraser afferma che un approccio volto a compensare le ingiustizie della distribuzione può finire per creare ingiustizie di riconoscimento (2008, p. 31).

La ridistribuzione affermativa presuppone una concezione universalistica del riconoscimento (uguale valore morale delle persone; intitolato da Fraser $(2008,2003$, 2002) come impegno formale al riconoscimento). Tuttavia, la pratica della ridistribuzione affermativa, se prolungata, tende a mettere in moto una dinamica secondaria di stigmatizzazione del riconoscimento che contraddice il proprio impegno formale per l'universalism ${ }^{[4]}$. L'autore (2008), in contrasto con questa logica con rimedi trasformativi per le ingiustizie distributive di classe, prevede che combinano programmi di assistenza sociale universalistici, tasse elevate, politiche macroeconomiche volte a creare la piena occupazione, un vasto settore pubblico non di mercato, importanti proprietà pubbliche e/o collettive e decisioni democratiche in materia di priorità socioeconomiche di base. I rimedi di redistribuzione trasformativa tendono a dissolvere la differenziazione di classe, riducendo le disuguaglianze sociali senza creare classi stigmatizzate di persone vulnerabili viste come beneficiarie di particolare generosità. Fraser afferma che questi rimedi tendono a promuovere la reciprocità e la solidarietà nei rapporti di riconoscimento, potendo così affrontare il risarcimento delle ingiustizie di ridistribuzione per aiutare, anche, a compensare alcune ingiustizie di riconoscimento (2008, p.31-33)

Fraser (2008) afferma che, come la ridistribuzione affermativa, la ridistribuzione trasformativa (in generale) presuppone una concezione universalistica del riconoscimento: pari valore morale delle persone. Tuttavia, a differenza della ridistribuzione affermativa, la pratica della ridistribuzione trasformativa tende a non sciogliere questa concezione.

Da questa discussione, dopo aver considerato solo i casi tipici-idee pure ad entrambe le estremità dello spettro concettuale, e contrastare gli effetti divergenti dei rimedi affermativi e trasformativi alle ingiustizie distributive radicate economicamente - da un 
lato - e quelli di riconoscimento culturalmente radicato - dall'altro - si vede che: 1 . i rimedi affermativi tendono generalmente a promuovere la differenziazione di gruppo, mentre i rimedi trasformativi tendono a destabilizzare e offuscare questa differenziazione; e 2. I rimedi di ridistribuzione affermativa possono suscitare una protesta di disprezzo, mentre i rimedi di ridistribuzione trasformativa possono contribuire a compensare alcune forme di non riconoscimento. In questo senso, Fraser $(2008,2003,2002)$ suggerisce un modo per riformulare il dilemma della ridistribuzionericonoscimento.

Tuttavia, nel concludere questa discussione, Fraser solleva l'interrogatole:

[...] Per quanto riguarda i gruppi sottoposti a entrambi i tipi di ingiustizia, quale sarebbe la combinazione di rimedi che funzionerebbero meglio per ridurre al minimo (se non eliminare per sempre) le interferenze reciproche che sorgono quando si cerca la ridistribuzione e il riconoscimento al stesso tempo? (2008, p. 33, la mia traduzione).

E questo dovrebbe ancora essere problematizzato.

\section{CONSIDERAZIONI FINALI: L'IL'AL-UMILATION DELLA FRASEANO DEBATE Alla REALIUM DI HIGHER EDUCATION IN BRASILE}

Si afferma che il Brasile, perché è caratterizzato da numerose disuguaglianze, di ordini diversi, è contrassegnato come un modello paradigmatico per pensare all'applicazione della teoria fraseane. In questo senso, le teorizzazioni di Fraser sono proposte per l'analisi dello scenario educativo brasiliano.

Come sostiene Ferreira (2010), la modernità, al suo arrivo in Brasile, ha trovato un paese con marchi di conservatorismo e tradizionalismo - una situazione che, in un certo senso, rimane ancora oggi in vari settori sociali. In Brasile, il pregiudizio e l'esclusione razziale sono avvenuti (e conservano ancora, in una certa misura) in modo velato, disistituzionale e apertamente riconosciuto. Ciò compromette, in molti casi, i processi di intervento del governo. Azioni come le politiche di azione affermativa sono ripetutamente viste come un affronto alle pari opportunità - anche se sono visibili disuguaglianze, segregazione e pregiudizio (FERREIRA, 2010). 
È in questo senso che i risultati delle autorità pubbliche al fine di ridurre le disuguaglianze sociali possono essere compresi dalle teorizzazioni di Fraser (2008, $2003,2002,2000)$ sul riconoscimento e la ridistribuzione. È essenziale che le politiche di ridistribuzione e riconoscimento non si contraddicano e, inoltre, siano guidate dal rispetto reciproco (APPLE, 2017, p. 32).

Le politiche di azione affermativa fanno parte di Fraser (2008, 2003, 2002), come rimedio contro le ingiustizie sociali. Tuttavia, come prevede Ferreira (2012), un punto importante da considerare in questa discussione si riferisce al fatto che, ancora da una prospettiva fraseana, le azioni affermative non sarebbero garanzie assolute per modificare positivamente la struttura che genera disuguaglianze. Questo perché, forse, a priori, riconoscono solo la necessità di distribuire a i gruppi storicamente compromessi, ma non risolvono necessariamente le impasse strutturali che generano tali disuguaglianze. L'aspettativa sarebbe che coloro che beneficiano di queste politiche possano - su larga scala e a lungo termine - trasmettere i loro risultati di crescita socioeconomica e culturale in modo intergenerazionale in modo che i loro discendenti non necessitino più di politiche di questo tipo di rimanere in aumento o raggiungere livelli di reddito e di status simili a quelli della popolazione che storicamente hanno ricevuto questi diritti in Brasile.

\section{RIFERIMENTI}

APPLE, Michael W. A educação pode mudar a sociedade?. Petrópolis: Vozes, 2017.

APPLE, Michael W.; BALL, Stephen J.; GANDIN, Luís Armando. Mapeamento da sociologia da educação: contexto social, poder e conhecimento. In: Sociologia da Educação: análise internacional. Michael W. Apple; Stephen J. Ball; Luís Armando Gandin (orgs.). Porto Alegre: Penso, 2013.

LIMA, Márcia. Ações afirmativas no governo Lula. In: Revista Novos Estudos. 2010.

FERREIRA, Wallace. Justiça e reconhecimento em Nancy Fraser. Teresina: Jus Navigandi, 2012. 
FRASER, Nancy. From Redistribution to Recognition? Dilemmas of Justice in a "Postsocialist" Age. In. Adding Insult to Injury. Nancy Fraser debates her critics. Edinburgh: Verso, 2008.

Justice Interruptus: Critical Reflections on the "Postsocialist" Condition. London: Routledge, 1997.

Redistribuição ou reconhecimento? Classe e status na sociedade contemporânea. In: Interseções - Revista de Estudos Interdisciplinares. UERJ, ano 4, n.1, 2002.

Recognition without Ethics? Theory, Culture \& Society, v. 18, p. 21-42, 2001.

Da redistribuição ao reconhecimento? Dilemas da justiça na era pós-socialista. In: Democracia hoje: novos desafios para a teoria democrática contemporânea. Jessé Souza (org.). Brasília: Editora Universidade de Brasília, 2000.

FRASER, N.; HONNETH, A. Redistribution or recognition?: a political philosophical exchange. London; New York: Verso, 2003.

GILLBORN, David; LADSON-BILLINGS, Gloria. Educação e Teoria Racial Crítica. In: Sociologia da Educação: análise internacional. Michael W. Apple; Stephen J. Ball; Luís Armando Gandin (orgs.). Porto Alegre: Penso, 2013.

HEILBORN, M. L.; ARAÚJO, L.; BARRETO, A. (Orgs). Gestão de Políticas Públicas em Gênero e Raça: GPP-GeR, Módulo I. Rio de Janeiro: CESPE; Brasília: Secretaria de Políticas Públicas para as Mulheres. 2010.

PEREIRA, Elisabete Monteiro de Aguiar. A universidade da modernidade nos tempos atuais. In: Revista Avaliação, v. 14, n. 1, p. 29-52. Campinas, 2009.

ZONINSEIN, Jonas; FERES JÚNIOR, João. Ação Afirmativa e Desenvolvimento. In: FERES JÚNIOR, João \& ZONINSEIN, Jonas (Orgs.). Ação Afirmativa e Universidade: experiências nacionais comparadas. Brasília: Editora Universidade de Brasília, 2006. 
2. Toma-se, aqui, como referência principal para a análise que se propõe, a seguinte obra: FRASER, Nancy. From Redistribution to Recognition? Dilemmas of Justice in a "Postsocialist" Age. In. Adding Insult to Injury. Nancy Fraser debates her critics. Edinburgh: Verso, 2008.

3. "People of color", nos termos de Fraser.

4. Nos termos de Fraser, essa dinâmica secundária (estigmatizante) pode ser entendida como o efeito de reconhecimento prático da redistribuição afirmativa (2008, p. 31-32).

Inviato: Ottobre, 2019.

Approvato: novembre 2019. 